\title{
Historical Cohort Study of the Efficacy and Safety of Piperacillin/Tazobactam versus Fourth-Generation Cephalosporins for Empirical Treatment of Febrile Neutropenia in Patients with Hematological Malignancies
}

\author{
Takashi Saito $^{1,3}$, Tatsuo Ichinohe ${ }^{2}$, Junya Kanda ${ }^{2}$, Miki Nagao ${ }^{1}$, Shunji Takakura ${ }^{1}$, Yutaka Ito ${ }^{1}$, \\ Yoshitsugu Iinuma ${ }^{1}$, Kouhei Yamashita ${ }^{2}$, Tadakazu Kondo ${ }^{2}$, Takayuki Ishikawa ${ }^{2}$, \\ Takashi Uchiyama $^{2,4}$, Satoshi Ichiyama ${ }^{1}$
}

\begin{abstract}
${ }^{1}$ Department of Infection Control and Prevention, Kyoto University Hospital, Kyoto, Japan; ${ }^{2}$ Department of Hematology and Oncology, Kyoto University Hospital, Kyoto, Japan; ${ }^{3}$ Department of Infection Control and Prevention, Shiga Medical Center for Adults, Shiga, Japan; ${ }^{4}$ Kitano Hospital, The Tazuke Kofukai Medical Research Institute, Osaka, Japan.

Email: t_saitoh@shiga-mca.jp
\end{abstract}

Received October $20^{\text {th }}, 2010$; revised December $5^{\text {th }}, 2010$; accepted December $10^{\text {th }} 2010$.

\begin{abstract}
We retrospectively evaluated the efficacy and safety of the combination drug piperacillin/tazobactam (PIPC/TAZ) in comparison with those of fourth-generation cephalosporins (4th Cephs) as initial empirical treatment in hematological malignancies patients with febrile neutropenia (FN). Among 200 patients assessed in this study, 49 had received PIPC/TAZ and 151 4th Cephs. Patient background characteristics were comparable between the two treatment groups. The overall efficacy rate in those receiving 4th Cephs and PIPC/TAZ was 57.0\% (86/151 patients) and 59.2\% (29/49 patients), respectively, with no significant difference detected between the two treatment regimens $(P=0.78)$. Treatment did not need to be discontinued or interrupted due to development of adverse drug reactions in any of the patients. Therefore in this study the efficacy and safety of PIPC/TAZ as initial antimicrobial treatment for FN in patients with hematological malignancies were not inferior to those of 4th Cephs. Based on the preliminary data obtained in this study, we propose to conduct a multicenter, prospective, controlled study to compare PIPC/TAZ versus CFPM given as empirical antimicrobial treatment against FN in patients with hematological malignancies.
\end{abstract}

Keywords: Febrile Neutropenia, Piperacillin/Tazobactam, Fourth-Generation Cephalosporins, Safety, Efficacy

\section{Introduction}

Hematological malignancies such as acute leukemia, myelodysplastic syndromes (MDS), malignant lymphoma, and multiple myeloma are often complicated by fever associated with decreases of neutrophil counts caused by anticancer drug treatment. As such, febrile neutropenia (FN) requires prompt treatment with broad-spectrum antimicrobials since it may be associated with life-threatening infections.

The Infectious Diseases Society of America (IDSA) recommends as initial treatment in patients with $\mathrm{FN}$ who are at high risk of serious infections either monotherapy with a third-generation cephalosporins, a fourth Genera- tion cephalosporins (4 th Ceph; cefepime [CFPM]), or a carbapenem or dual therapy with an aminoglycoside plus an antipseudomonal penicillin (such as in the combination drug piperacillin/tazobactam; PIPC/TAZ), CFPM, ceftazidime, or carbapenem [1]. The 2007 National Comprehensive Cancer Network (NCCN) Prevention and Treatment of Cancer-Related Infections in Clinical Practice Guidelines in Oncology [2] recommend PIPC/TAZ and place the same emphasis on monotherapy with a third - or fourth-generation cephalosporins (ceftazidime or CFPM) or a carbapenem (imipenem/cilastin or meropenem) as the IDSA guidelines. The Japanese [3] and German [4] guidelines are also mostly consistent with the 
IDSA guidelines.

The efficacy and safety of PIPC/TAZ given as initial treatment against FN have so far not been reported in Japanese patients. Therefore in this historical cohort study we evaluated and compared the efficacy and safety of PIPC/TAZ with those of 4th Cephs in the setting of initial antimicrobial treatment for $\mathrm{FN}$, as a preliminary step to our conducting a future controlled study investigating the usefulness of PIPC/TAZ in patients with FN secondary to hematological malignancies.

\section{Patients and Methods}

\subsection{Patients}

Patients with hematological malignancies who were admitted to the Department of Hematology and Oncology at Kyoto University Hospital between January 2005 and July 2006, treated with anticancer drugs and/or transplantation, and subsequently administered either a 4th Cephs (CFPM or cefozopran) or PIPC/TAZ as initial treatment against FN were included in this study. Treatment was given on an inpatient basis. Before initiation of antibiotic therapy, blood samples for cell cultures were obtained from a peripheral vein in the context of fever and/or other signs consistent with infection. Data on specific site infections were not collected. Prophylactic antimicrobials, including quinolones, had not been used in any of the patients. This study was approved by the Ethics Committee of Kyoto University Graduate School and the Faculty of Medicine.

\subsection{Antimicrobial Treatment}

The daily dosage of the 4th Cephs was $4 \mathrm{~g}$, with the drug administered in three divided doses $(1 \mathrm{~g}$ each at 09:00 and 15:00; $2 \mathrm{~g}$ at 21:00), and that of PIPC/TAZ was 13.5 $\mathrm{g}$ also administered intravenously in three divided doses (PIPC $4 \mathrm{~g} / \mathrm{TAZ} 500 \mathrm{mg}$ each at 9:00, 15:00, and 21:00). The management after empiric antibiotic therapy was conducted according to the algorithm recommended by the IDSA guidelines [1]. An aminoglycoside was used concomitantly in both treatment groups at the discretion of the attending hematologists. None of the patients required adjustment of the antimicrobial drug dose or the dosing interval on account of renal dysfunction.

\subsection{Study Parameters}

Data for the analysis included age, sex, underlying disease, type of transplant, type of initial treatment against FN, baseline neutrophil count (at the start of initial treatment), treatment switch, and duration of neutrophil count $<$ $100 / \mathrm{mm}^{3}$ or $<500 / \mathrm{mm}^{3}$ in each patient.

FN was defined as an axillary temperature $\geq 37.5^{\circ} \mathrm{C}$ with a neutrophil count $<500 / \mathrm{mm}^{3}$. Thermometry was performed $\geq 3$ times daily: in the morning and afternoon and before going to bed, according to the condition of each individual patient. Potential noninfectious causes of fever were not ruled out in this study. Patients treated successfully with the initial treatment alone were defined as "responders" whereas the remainder comprised the "nonresponders" group. That is, efficacy of the study drugs was assessed based on whether symptoms of FN were resolved by the initial empiric therapy or the patients were switched to other antimicrobial agents.

All adverse drug reactions were recorded on central database.

\subsection{Statistical Analysis}

Student's $t$-test was used to analyze the influence of age and duration of neutrophil suppression. Chi-square test or Fisher's exact test was used to analyze the influence of sex, underlying disease, and type of transplant and efficacy rate.

\section{Results}

\subsection{Patient Characteristics}

Of the 200 patients included in this study, 151 were treated with 4th Cephs and 49 received PIPC/TAZ (P/T group) as initial empirical treatment against FN. None of the patients died during the study, which was conducted for $\leq 30$ days of treatment. The patient characteristics are presented in Table 1. Seventy-eight patients in the 4th Cephs group (52\%) and 30 patients in the $\mathrm{P} / \mathrm{T}$ group $(55 \%)$ had acute leukemia or MDS $(P=0.67)$. Twenty-two patients in the 4 th Cephs group (15\%) and 8 patients in the $\mathrm{P} / \mathrm{T}$ group $(16 \%)$ had undergone transplantation of hematopoietic progenitor cells $(P=0.76)$. All patients in both groups had neutrophil counts < $500 / \mathrm{mm}^{3}$ at the start of treatment. Furthermore, 45 patients in the 4th Cephs group (30\%) and 14 patients in the $\mathrm{P} / \mathrm{T}$ group (29\%) had neutrophil counts $<100 / \mathrm{mm}^{3}$ at the start of treatment $(P=0.87)$. The mean duration of having a neutrophil count $<100 / \mathrm{mm}^{3}$ and $<500 / \mathrm{mm}^{3}$ was comparable between the two treatment groups $(P=0.23$ and 0.60 , respectively). Forty-two patients in the 4th Cephs group (28\%) and 12 patients in the $\mathrm{P} / \mathrm{T}$ group $(24 \%)$ were treated concurrently with an aminoglycoside $(P=0.65)$.

Etiologic organisms obtained from blood culture examinations were positively identified in 11 patients in the 4th Cephs group (Esherichia coli, $\mathrm{n}=3$; Klebsiella pneumoniae, $\mathrm{n}=3$; Pseudomonas aeruginosa, $\mathrm{n}=2$; Enterobacter cloacae, $\mathrm{n}=1$; Klebsiella oxytoca, $\mathrm{n}=1$; Streptococcus 
20 Historical Cohort Study of the Efficacy and Safety of Piperacillin/Tazobactam versus Fourth-Generation Cephalosporins forEmpirical Treatment of Febrile Neutropenia in Patients with Hematological Malignancies

mitis, $\mathrm{n}=1$ ) and in four patients in the $\mathrm{P} /$ T group (E. coli, $\quad \mathrm{n}=3$; Streptococcus viridans, $\mathrm{n}=1$ ).

Table 1. Baseline characteristics of patients with febrile neutropenia.

\begin{tabular}{lccc}
\hline Parameter & $\begin{array}{c}\text { 4th Cephs group } \\
(n=151)\end{array}$ & $\begin{array}{c}\text { P/T group } \\
(n=49)\end{array}$ & $P$-value \\
\hline Mean age (range), years & $52.1(18-81)$ & $52.8(24-74)$ & 0.77 \\
Sex, M/F & $67 / 84$ & $25 / 24$ & 0.42 \\
Underlying disease, $n$ (\%) & & & \\
Acute leukemia and MDS & $78(52)$ & $30(61)$ & 0.24 \\
Malignant lymphoma & $46(30)$ & $15(22)$ & 0.98 \\
Multiple myeloma & $14(9)$ & $2(4)$ & 0.37 \\
Chronic leukemia & $10(7)$ & 0 & 0.12 \\
Immunoblastic lymphadenopathy & $2(1)$ & $1(2)$ & 0.57 \\
Aplastic aplasia & $1(1)$ & 0 & 0.99 \\
Plasmacytoma & 0 & $1(1)$ & 0.25 \\
Transplantation, $n(\%)$ & $22(15)$ & $8(16)$ & 0.76 \\
Myeloablative & $10(7)$ & $4(8)$ & 0.75 \\
Nonmyeloablative & $8(5)$ & $2(4)$ & 0.99 \\
Autologous & $4(3)$ & $2(4)$ & 0.64 \\
Neutrophil count, $n(\%)$ & & & \\
$<100 /$ mm $^{3}$ & $45(30)$ & $14(29)$ & 0.87 \\
100-500/mm & $106(70)$ & $35(71)$ & 0.87 \\
Mean (SD) duration of neutropenia, days & & & \\
$<100 / \mathrm{mm}^{3}$ & $9.9(8.3)$ & $11.9(9.5)$ & 0.23 \\
$<500 / \mathrm{mm}^{3}$ & $12.6(10.5)$ & $13.5(10.3)$ & 0.60 \\
Dual therapy, $n$ (\%) & $42(28)$ & $12(24)$ & 0.65 \\
\hline
\end{tabular}

4th Cephs: fourth-generation cephalosporins, $\mathrm{P} / \mathrm{T}$ : piperacillin/tazobactam.

Table 2. Efficacy rate of fourth-generation cephalosporins or piperacillin/tazobactam as initial empirical therapy in patients by subgroup.

\begin{tabular}{lccc}
\hline & \multicolumn{2}{c}{ Efficacy rate, $n(\%)$} & P-value \\
\cline { 2 - 3 } & $\begin{array}{c}\text { 4th Cephs group } \\
(n=151)\end{array}$ & $\begin{array}{c}\text { P/T group } \\
(n=49)\end{array}$ & \\
\hline Total & $86 / 151(57.0)$ & $29 / 49(59.2)$ & 0.78 \\
Underlying disease & & & \\
$\quad$ Acute leukemia and MDS & $39 / 78(50.0)$ & $17 / 30(56.7)$ & 0.53 \\
$\quad$ Other hematological disorders & $47 / 73(64.4)$ & $12 / 19(63.2)$ & 0.92 \\
Transplantation & & & 0.70 \\
$\quad$ Yes & $9 / 22(40.9)$ & $4 / 8(50.0)$ & 0.88 \\
$\quad$ No & $77 / 129(59.7)$ & $25 / 41(61.0)$ & 0.99 \\
Monotherapy & $65 / 109(59.6)$ & $22 / 37(59.5)$ & 0.61 \\
Dual therapy & $21 / 42(50.0)$ & $7 / 12(58.3)$ & \\
\hline
\end{tabular}

4th Cephs: fourth-generation cephalosporins, P/T: piperacillin/tazobactam.

\subsection{Clinical Efficacy}

Efficacy rates of the two test agents given as initial empiric therapy against FN are presented in Table 2 . In the 4th Cephs and $\mathrm{P} / \mathrm{T}$ groups, the overall efficacy rate was $57.0 \%$ (86/151 patients) and 59.2\% (29/49 patients), respectively, with no significant difference detected between the two groups $(P=0.78)$. Moreover, the difference of efficacy rate was not statistically significant between acute leukemia and MDS patients in the 4th Cephs group (50.0\% [39/78 patients]) and $\mathrm{P} / \mathrm{T}$ group $(56.7 \%$ [17/30 patients]; $P=0.54$ ) and in those with other hematological disorders $(64.4 \%$ [ $47 / 73$ patients] and $63.2 \%$ [12/19 patients], respectively; $P=0.92$ ). Furthermore, the between-group efficacy rate was not different in posttransplant patients $(40.9 \%$ [9/22 patients] and 50.0\% [4/8 patients], respectively; $P=0.70$ ) and those without transplantation $(59.7 \%$ [77/129 patients] and $61.0 \%$ [25/41 patients], respectively; $P=0.88$ ). The efficacy rate in patients receiving monotherapy was $59.6 \%$ 
$(65 / 109)$ in the 4 th Cephs group and $59.5 \%(22 / 37$ patients) in the $\mathrm{P} / \mathrm{T}$ group $(P=0.99)$ whereas in those concomitantly receiving an aminoglycoside the rate was $50.0 \%$ (21/42 patients) and 58.3\% (7/12 patients), respectively $(P=0.61)$.

\subsection{Adverse Drug Reactions}

No adverse drug reaction requiring discontinuation or switching of the study treatments was noted in any of the patients.

\section{Discussion}

According to the Japan Adult Leukemia Study Group's Supportive Therapy Subcommittee questionnaire survey of 196 participating institutions nationwide, cephalosporin \pm aminoglycoside, carbapenem \pm aminoglycoside, and antipseudomonal penicillin \pm aminoglycoside were used as the initial treatment for $\mathrm{FN}$ in $51 \%, 23 \%$, and $11 \%$ of the responding institutions, respectively [5]. The 2002 IDSA guidelines and recently published Japanese guidelines for antimicrobial therapy against $\mathrm{FN}$ recommend monotherapy with a broad-spectrum cephalosporin or carbapenem, combination of both these drug classes, or antipseudomonal penicillin and an aminoglycoside $[1,3]$. Increasing emergence of multidrug-resistant Pseudomonas has become a clinical problem in recent years [6-10]. Concomitant use of carbapenems with cancer chemotherapy is a potential risk factor for the development of multidrug-resistant Pseudomonas infection [11]. We consider PIPC/TAZ as an alternative to carbapenems because of these two medications' comparable antimicrobial spectrums.

In the present study, the overall efficacy rate was $57.0 \%$ in the 4th Cephs group and $59.2 \%$ in the $\mathrm{P} / \mathrm{T}$ group. Similar to our findings, the efficacy rates of 4 th Cephs \pm aminoglycoside and PIPC/TAZ \pm aminoglycoside in cases of $\mathrm{FN}$ reported from previous controlled studies varied at $21-62 \%$ and $27 \%-61 \%$, respectively [12-15]. This variability in the efficacy rates noted in these studies is likely related to differences in the definition of efficacy, which was variously set as 2- or 3-day defervescence, microbiological eradication, test of cure, and so on.

Precise indications for the concurrent use of aminoglycosides in the initial treatment against $\mathrm{FN}$ remain controversial. There is no reference to this issue in the IDSA guidelines, while the Japanese guidelines recommend concurrent use of an aminoglycoside as an option in patients receiving induction therapy for acute leukemia and those undergoing hematopoietic stem cell transplantation $[1,3]$. As in previous studies, $20-25 \%$ of patients included in the present study received concurrent admini- stration of an aminoglycoside with one of the guideline-recommended drugs as initial treatment. One previous study reported a higher efficacy rate in patients receiving adjuvant aminoglycoside than in those receiving monotherapy [16]. In the present study, on the other hand, the efficacy rate in patients receiving concurrent aminoglycoside was slightly lower compared with that in patients on monotherapy in both treatment groups, although the difference was not statistically significant $(P$ $=0.29$ and 0.95 in the 4 th Cephs and $\mathrm{P} / \mathrm{T}$ groups, respectively). Use of dual therapy in many posttransplant patients and in patients with acute leukemia/MDS may be the reason for the lower efficacy rate associated with concurrent aminoglycoside administration in our study.

One of the main objectives of this study was to determine whether the efficacy of PIPC/TAZ is comparable to that of 4 th Cephs as initial treatment against $\mathrm{FN}$, as a preliminary assessment of the feasibility of our conducting a future prospective controlled study in this setting. The main limitations of this study were its non-prospective design and the efficacy evaluation being not solely based on fever reduction as in previous Japanese studies $[16,17]$. Here, treatment efficacy was evaluated based on the need for switching antimicrobial drugs, because this was determined by attending hematologists on the basis of comprehensive assessments of clinical symptoms, laboratory tests, and radiological findings in individual patients. The third limitation of this study is that the antimicrobials were given to the patients at 9:00, 15:00, and 21:00 hours, taking into account the patients' sleeping times and the nursing shifts. As reported elsewhere, intravenous antimicrobial infusion should desirably be given at 8-hour intervals according to the antimicrobial pharmacokinetics/pharmacodynamics [12-15].

We found that the efficacy and safety of PIPC/TAZ given as initial treatment against $\mathrm{FN}$ were not inferior to those of 4th Cephs. Based on the preliminary data obtained in this study, we propose to conduct a multicenter, prospective, controlled study to compare PIPC/TAZ versus CFPM given as empirical antimicrobial treatment against $\mathrm{FN}$ in patients with hematological malignancies.

This study was presented, in part, at the 54th Japanese Society of Chemotherapy West Japan Branch Conference and was awarded the 1st Japanese Society of Chemotherapy Head of West Japan Branch Award (Clinical Division).

\section{REFERENCES}

[1] W. T. Hughes, D. Armstrong, G. P. Bodey, E. J. Bow, A. E. Brown, T. Calandra, et al., "Guidelines for the Use of Antimicrobial Agents in Neutropenic Patients with Cancer," Clinical Infectious Diseases, Vol. 34, No. 6, 2002, 
pp. 730-751. doi:10.1086/339215

[2] NCCN Clinical Practice Guideline in Oncology ${ }^{\mathrm{TM}} 2007$, "Prevention and Treatment of Cancer-Related Infections VI 2007 Initial Therapy for Fever and Neutropenia FEV1," 2007.http://www.nccn.org/professionals/physician_gls/P DF/fever.pdf

[3] T. Masaoka, "Evidence-Based Recommendations for Antimicrobial Use in Febrile Neutropenia in Japan: Executive Summary," Clinical Infectious Diseases, Vol. 39, Suppl 1, 2004, pp. S49-52. doi:10.1086/383054

[4] A. Glasmacher and M. von Lilienfeld-Toal, "An Evidence Based Review of the Available Antibiotic Treatment Options For Neutropenic Patients and a Recommendation for Treatment Guidelines," International Journal of Infectious Diseases, Vol. 10, Suppl 2, 2006, pp. S9-16. doi:10.1016/S1201-9712(06)60003-9

[5] M. Yoshida and R. Ohno, "Current Antimicrobial Usage for the Management of Infections in Leukemic Patients in Japan: Results of a Survey," Clinical Infectious Diseases, Vol. 39, Suppl 1, 1995, pp. S11-14. doi:10.1086/383044

[6] D. R. Burwen, S. N. Banerjee, and R. P. Gaynes, "Ceftazidime Resistance among Selected Nosocomial Gram-Negative Bacilli in the United States," Journal of Infectious Diseases, Vol. 170, No. 6, 1994, pp. 1622-1625.

[7] A. C. Basustaoglu, H. Gun, M. A. Saraçli, M. Baysallar and T. Haznedaroglu, "Development of Resistance to Imipenem among Nosocomial Isolates of Pseudomonas Aeruginosa," European Journal of Clinical Microbiology and Infectious Diseases, Vol. 14, No. 5, 1995, pp. 469-470. doi:10.1007/BF02114909

[8] N. Troillet, M. H. Samore and Y. Carmeli, "ImipenemResistant Pseudomonas Aeruginosa: Risk Factors and Antibiotic Susceptibility Patterns", Clinical Infectious Diseases, Vol. 25, No. 5, 1995, pp. 1094-1098. doi:10.1086/516092

[9] National Nosocomial Infections Surveillance System, "National Nosocomial Infections Surveillance System Report, data summary from January 1992 to June 2002, issued August 2002," American Journal of Infection Control, Vol. 30, No. 8, 2002, pp. 458-475. doi:10.1067/mic.2002.130032

[10] E M D'Agata, "Rapidly Rising Prevalence of Nosocomial Multidrug-Resistant, Gram-Negative Bacilli: A 9-Year Surveillance Study," Infection Control and Hospital Epidemiology, Vol. 25, No. 10, 2004, pp. 842-846.
[11] N. Ohmagari, H. Hanna, L. Graviss, B. Hackett, C. Perego, V. Gonzalez, et al., "Risk Factors for Infections with Multidrug-Resistant Pseudomonas Aeruginosa in Patients with Cancer," Cancer, Vol. 104, No. 1, 2005, pp. 205-212. doi:10.1002/cncr.21115

[12] A. Bohme, P. M. Shah, W. Stille and D. Hoelzer, "Piperacillin/Tazobactam Versus Cefepime as Initial Empirical Antimicrobial Therapy in Febrile Neutropenic Patients: A Prospective Randomized Pilot Study," European journal of medical research, Vol. 3, No. 7, 1998, pp. 324-330.

[13] F. Bauduer, T. Cousin, O. Boulat, F. Rigal-Huguet, L. Molina, N. Fegueux, et al., "A Randomized Prospective Multicentre Trial of Cefpirome versus Piperacillin-Tazobactam in Febrile Neutropenia," Leukemia Lymphoma, Vol. 42, No. 3, 2001, pp. 379-386. doi:10.3109/10428190109064594

[14] M. A. Sanz, J. López, J. J. Lahuerta, M. Rovira, M. Batlle, C. Pérez, et al., "Cefepime Plus Amikacin Versus Piperacillin-Tazobactam Plus Amikacin for Initial Antibiotic Therapy in Haematology Patients with Febrile Neutropenia: Results of an Open, Randomized, Multicentre Trial," Journal of antimicrobial chemotherapy, Vol. 50, No. 1, 2002, pp. 79-88. doi:10.1093/jac/dkf087

[15] E. J. Bow, C. Rotstein, G. A. Noskin, M. Laverdiere, A. P. Schwarer, B. H. Segal, et al., "A Randomized, Open-Label, Multicenter Comparative Study of the Efficacy and Safety of Piperacillin-Tazobactam and Cefepime for the Empirical Treatment of Febrile Neutropenic Episodes in Patients with Hematologic Malignancies," Clinical Infectious Diseases, Vol. 43, Suppl 1, 2006, pp. 447-459.doi:10.1086/505393

[16] K. Tamura, K. Imajo, N. Akiyama, K. Suzuki, A. Urabe, K. Ohyashiki, et al., "Randomized Trial of Cefepime Monotherapy or Cefepime in Combination with Amikacin as Empirical Therapy for Febrile Neutropenia," Clinical Infectious Diseases, Vol. 39, Suppl 1, 2004, pp. S15-24.doi:10.1086/383046

[17] K. Tamura, H. Matsuoka, J. Tsukada, M. Masuda, S. Ikeda, E. Matsuishi, et al., "Cefepime or Carbapenem Treatment for Febrile Neutropenia as a Single Agent Is as Effective as a Combination of 4th-Generation Cephalosporin + Aminoglycosides: Comparative Study," American Journal of Hematology, Vol. 71, No. 4, 2002, pp. 248-255. doi:10.1002/ajh.10236 